\title{
Psychothérapie et science : un nombrilisme ?
}

\author{
Gerhard Burda
}

Psychotherapie-Wissenschaft 9 (2) 412019

www.psychotherapie-wissenschaft.info

CC BY-NC-ND

https://doi.org/10.30820/1664-9583-2019-2-41

Mots clés : science de la psychothérapie, théorie scientifique, science, sujet, différence de soi-même

Le texte poursuit deux buts : d'abord d'explorer la question de savoir comment la science de la psychothérapie peut être qualifiée de science à part entière. Dans un deuxième temps est ouverte à la discussion la question de savoir si le "facteur subjectif » peut être concilié avec une notion de science générale - la plupart du temps informée en termes de sciences naturelles. Ces questions sont également abordées par Markus Erismann dans les deux textes «Réflexions de théorie scientifique par rapport à la science de la psychothérapie » (2016) et "La notion scientifique de la science de la psychothérapie " (dans ce cahier). En ce qui concerne le premier texte, je plaiderais en faveur de l'adoption d'une perspective de théorie scientifique externe. Erismann fait au contraire tout simplement du caractère pré-paradigmatique des orientations de la psychothérapie le paradigme des sciences psychothérapeutiques en soulignant l'autoréflexivité élevée des méthodes psychothérapeutiques. Cette approche est à mon avis circulaire, elle efface les lignes de justification ainsi que les notions de théorie scientifique, de science de la psychothérapie et de psychothérapie. Le second texte d'Erismann vise à faire ressortir une concordance entre la notion scientifique de science de la psychothérapie et une notion scientifique généralement reconnue. Il s'agit du rapport que les facteurs importants pour la psychothérapie que sont le subjectif, l'individuel et le contradictoire entretiennent avec des critères scientifiques généraux tels que l'objectivité, la possibilité de vérifier et l'intersubjectivité. Ce second texte se rattache étroitement au premier et à la problématique que le caractère scientifique est censé être généré à partir du nombrilisme des psychothérapies. J'essaye au contraire de montrer que ni les contradictions inhérentes (différences par rapport à soi) ni le "facteur subjectif " tant honni ne doivent être éliminés de la science. Différence par rapport à soi veut dire que chaque forme concrète d'identité contient toujours une certaine contradiction, une ambivalence, quelque chose de paradoxal, une contre-logique ou un dédoublement en soi. Cette circonstance n'épargne ni nos notions de science ou de sujet ni nos conceptions de science de la psychothérapie. On peut par exemple s'en apercevoir en constatant simplement que des sens conflictuels peuvent être attribués à n'importe quel objet scientifique, sens qui peuvent tous finalement être rattachés à l'arrière-plan fantasmatique de notre faculté cognitive.

\section{L'auteur}

Gerhard Burda est philosophe, scientifique spécialisé en psychothérapie et analyste didacticien à Vienne. Il est depuis de nombreuses années président de l'Österreichische Gesellschaft für Analytische Psychologie (société autrichienne de psychologie analytique) et membre de l'École Lacan de Vienne ainsi qu'auteur de nombreuses publications à la convergence de la philosophie et de l'analyse psychologique et médiatique ; dernières parutions : Psychoanalyse der Erlösung (2016) (Psychanalyse de la rédemption), Mediale Identität/en (2018) (Identité/s médiatique/s), Pandora und die Metaphysica medialis (2019) (Pandora et la Metaphysica medialis).

\section{Contact}

Mag. Dr. Dr. habil. Gerhard Burda

Penzinger Str. 69/11

1140 Wien, Austria

E-Mail : comger@gmx.at

www.gerhardburda.com 